\title{
Chaotic reconnection due to fast mixing of vortex-current filaments
}

\author{
Yuichi Yatsuyanagi ${ }^{1}$, Tadatsugu Hatori ${ }^{2}$, and Tomokazu Kato ${ }^{3}$ \\ ${ }^{1}$ RIKEN (The Institute of Physical and Chemical Research), Wako-shi, Saitama 351-0198, Japan \\ ${ }^{2}$ Faculty of Science, Kanagawa University, Hiratsuka-shi, Kanagawa 259-1293, Japan \\ ${ }^{3}$ Department of Applied Physics, Waseda University, Shinjuku-ku, Tokyo 169-8555, Japan
}

(Received May 19, 2000; Revised February 28, 2001; Accepted February 28, 2001)

\begin{abstract}
We propose a new reconnection mechanism "chaotic reconnection". A basic mechanism of the chaotic reconnection is examined by means of numerical simulations of collision between two vortex-current filaments. The term "reconnection" means a reconnection of the filaments. We conclude that thle chaotic process works to enhance the reconnection rate of the filaments. We shall propose a similar chaotic process as a candidate for the mechanism of the fast magnetic reconnection.
\end{abstract}

\section{Introduction}

Nowadays many researchers share a common aspect that the magnetic reconnection is a fundamental process in the solar flare (Shibata, 1996). It is accepted in most cases that the electric resistivity plays a minor role and most of the theoretical works on the magnetic reconnection to date deal with the time scale problem (Biskamp, 1997; Horiuchi et al., 1999).

We expect that the magnetic field has a chaotic configuration in the diffusion region. Thus we shall propose a new reconnection model, "chaotic reconnection". In our model, the diffusion region contains many vortex-current filaments with axial magnetic field. The configuration of the filaments may evolve into chaotic one with time. Although the direct approach to reveal the mechanism of our reconnection model enhanced by the chaotic process entails three-dimensional MHD simulation, it is difficult due to the high magnetic Reynolds number. Thus we chose a filament model and examine an interaction of the two vortex-current filaments in this paper. We consider that the essential mechanism of the chaotic reconnection may be demonstrated by the simple filament model.

In Section 2, we give simulation results of collision between two straight vortex-current filaments and diagnosis via the Lyapunov exponents. The collision means the strong interaction between two filaments and yields fast mixing of the filaments. The configuration of the filaments becomes chaotic after the collision.

In Section 3, we show the basic mechanism of the chaotic reconnection due to the fast mixing of the filaments. The mechanism is revealed by the evaluation of the reconnection rates. We conclude that the chaotic process works to enhance the reconnection rate of the filaments.

Copy right (C) The Society of Geomagnetism and Earth, Planetary and Space Sciences (SGEPSS); The Seismological Society of Japan; The Volcanological Society of Japan; The Geodetic Society of Japan; The Japanese Society for Planetary Sciences.

\section{Simulation Results}

We have introduced the vortex-current filament model in our previous papers (Yatsuyanagi et al., 1996, 1998). The vortex-current filament consists of the electric current and the vorticity inside it. As basic equations, we use the ideal MHD equations with gravity. Integrating the equation of motion over the small volume element, we obtain a macroscopic force balance equation correct to $O\left(\rho^{-2}\right)$ where $\rho$ is a local radius of curvature of the filament. The velocity of the filament $\partial \boldsymbol{R} / \partial t$ is given in the follwing form with the cutoff Biot-Savart integral correct to $\mathrm{O}\left(\rho^{-1}\right)$ (Yatsuyanagi et al., 1996):

$$
\begin{aligned}
\left(\frac{\partial \boldsymbol{R}}{\partial t}\right)_{\perp}= & -\frac{J}{\kappa}\left(\boldsymbol{B}_{\mathrm{E}}\right)_{\perp}+\left(\boldsymbol{u}_{\mathrm{E}}\right)_{\perp} \\
& +\frac{\mu_{0} J^{2}}{4 \pi \kappa} \int \frac{(\boldsymbol{R}-\boldsymbol{x}) \times \mathrm{d} \boldsymbol{x}}{\left(|\boldsymbol{R}-\boldsymbol{x}|^{2}+\alpha^{2} a^{2}\right)^{3 / 2}} \\
& -\frac{\kappa}{4 \pi} \int \frac{(\boldsymbol{R}-\boldsymbol{x}) \times \mathrm{d} \boldsymbol{x}}{\left(|\boldsymbol{R}-\boldsymbol{x}|^{2}+\beta^{2} a^{2}\right)^{3 / 2}} \\
& +\frac{\pi a^{2}}{\kappa} \boldsymbol{g} \times \boldsymbol{s}+O\left(\rho^{-2}\right) .
\end{aligned}
$$

Here the mass density is constant and normalized to unity. The scale lengh of the system is taken as $\hat{x}=a$ where $a$ is initial radius of the filament and initially normalized to unity in the simulations. The characteristic value of the magnetic field is taken as $\hat{B}$. The Alfvén velocity and time scale are given by $\hat{u}=\hat{B} / \sqrt{\mu_{0}}$ and $\hat{t}=\hat{x} / \hat{u}$, respectively. The characteristic values of the electric current density and velocity are denoted by $\hat{j}=\hat{B} / \hat{x} \mu_{0}$ and $\hat{\omega}=\hat{B} / \hat{x} \sqrt{\mu_{0}}$, respectively. In numerical simulations, we introduce dimensionless variables and all the above-mentioned quantities are utilized as scaling factors. It is noticeable that the ratio $\hat{\omega} / \hat{j}$ has a magnitude of $\sqrt{\mu_{0}} \approx 10^{-3}$.

Illustration of the initial conditions of the simulation is given in Fig. 1. The most crucial parameter in our simulations is symmetry of the initial configuration of the filaments. The symmetry of the initial configuration is determined by an 


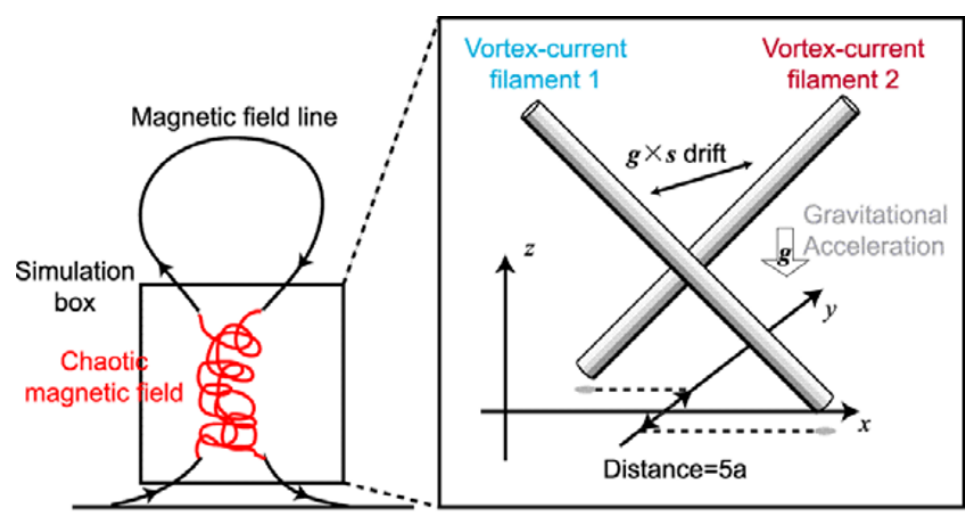

$\begin{array}{ll}\text { (a) Solar flare } & \text { (b) Simulation }\end{array}$

Fig. 1. Schematics of the simulations are shown.

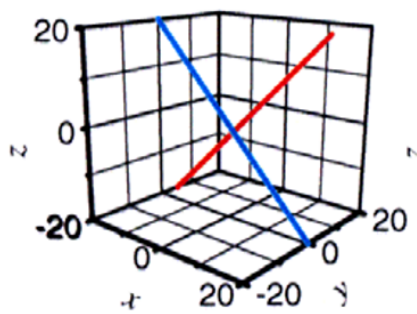

Type (a)

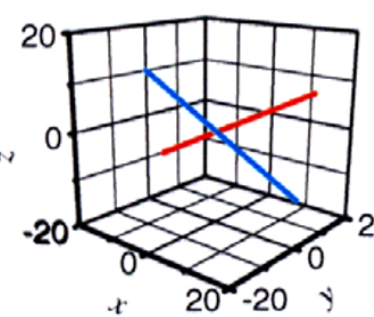

Type (b)

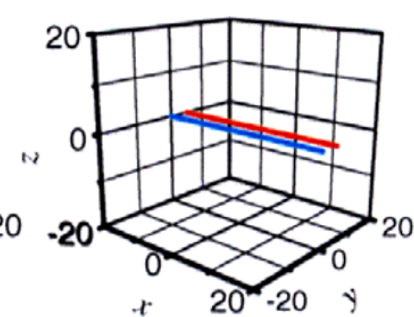

Type (c)

Fig. 2. Initial conditions of the types (a), (b) and (c) are shown.

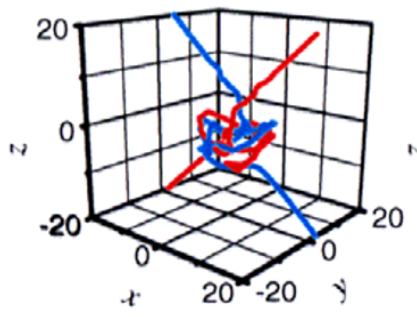

Type (a)

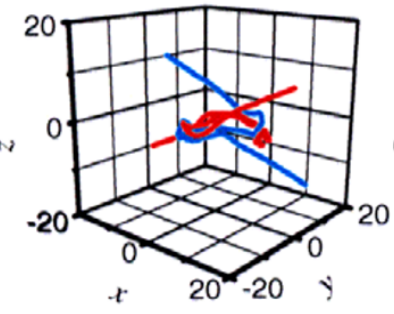

Type (b)

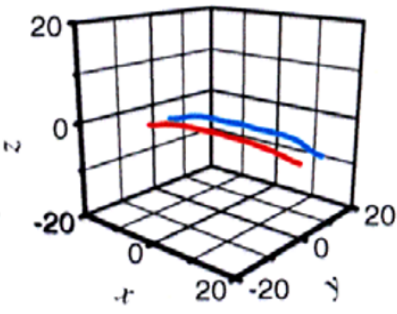

Type (c)

Fig. 3. Snap shots of the configuration of the filaments at $T=75 \times 10^{4} \Delta t$ are shown.

initial angle $\theta$ between the two filaments projected on the $x-z$ plane. The values of the initial angle are chosen as $\pi / 2$, $2 \arctan (1 / 2)$, and zero which are called types (a), (b), and (c), respectively as shown in Fig. 2. The most symmetrical case is $\theta=0$ and the most asymmetrical case is $\theta=\pi / 2$.

We show typical results of simulations in Fig. 3. For all the types, the filaments attract each other and then collide. Although the results for types (a) and (b) show complicated configuration near the collisional region, the result for type (c) shows non-complicated configuration. This is because the initial configurations of the types (a) and (b) are more asymmetrical than that of the type (c).

The time evolution of the instantaneous Lyapunov exponent is shown in Fig. 4. The largest peak for type (c) is due to the collision where the two filaments fully overlaps with

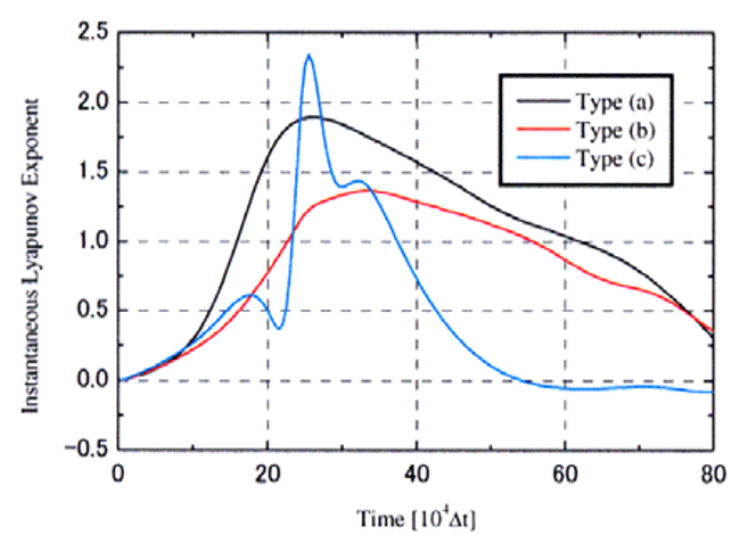

Fig. 4. Evolution of the instantaneous Lyapunov exponents is shown. 


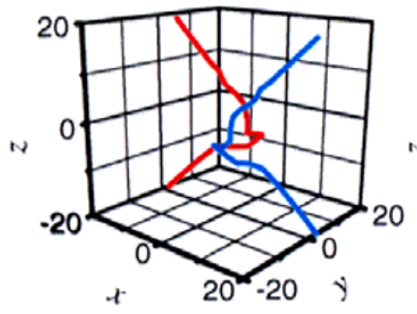

Type (a)

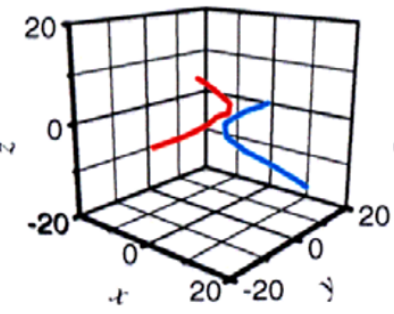

Type (b)

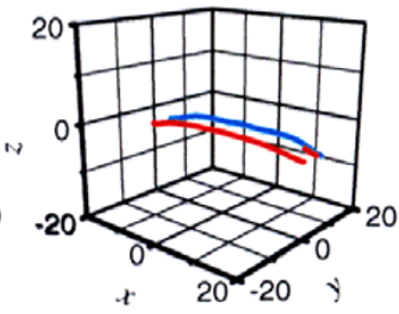

Type (c)

Fig. 5. Trajectory of the three-dimensional space-averaged distribution of the electric current is shown. Original data are given in Fig. 3.

each other. We consider that the other peaks at that time for types (a) and (b) are caused by the same reason. After the collision, the exponents only for types (a) and (b) are positive. We, therefore, conclude that the chaotic configuration is induced by the collision of the two filaments with initially low-symmetrical configuration.

\section{Chaotic Reconnection}

We consider when the filament 1 approaches the filament 2 and the electric currents inside the filaments are antiparallel to each other, the net electric current must be nearly zero. This means the filaments should locally annihilate each other in that region. Here we introduce a three-dimensional space averaging. The three-dimensional space averaging is a kind of mixing. It is well known that the mixing increases the entropy of the system, and this implies that the mixing is an irreversible process. Thus the space averaging should introduce a dissipation process into the system artificially. We calculate the three-dimensional space-averaged distribution of the electric currents numerically from the results shown in Fig. 3, and trace the trajectories by its distribution. The results are shown in Fig. 5.

For types (a) and (b) in Fig. 5, the macroscopic filaments, which mean the reconstructed filaments obtained by tracing the distribution of the electric current, are reconnected with each other. The reconnection is due to the chaotic configuration induced by the collision of the two filaments in lowsymmetry system. Thus it is obvious that the reconnection is not observed for type (c) because the initial configuration is symmetrical and the configuration does not evolve into a chaotic one. We call this reconnection mechanism "chaotic reconnection" from now on (Yatsuyanagi et al., 1999).

One can estimate the efficiency of the reconnection process by a reconnection rate. We assume the reconnection rate $R_{\mathrm{f}}(t)$ of the chaotic reconnection is given by the following form:

$$
R_{\mathrm{f}}(t)=D(t) \Omega(t, \theta),
$$

where $D(t)$ is a conventional (non-chaotic) reconnection rate in a single overlapping region where the dissipation plays an essential role. The notation $\Omega(t, \theta)$ is a non-dimensional factor and represents a normalized overlapping volume between the filaments per unit volume. The illustrations of the diffusion regions are shown in Fig. 6. The size of the diffusion region of the chaotic reconnection is relatively large compared with that of the traditional reconnection theory.

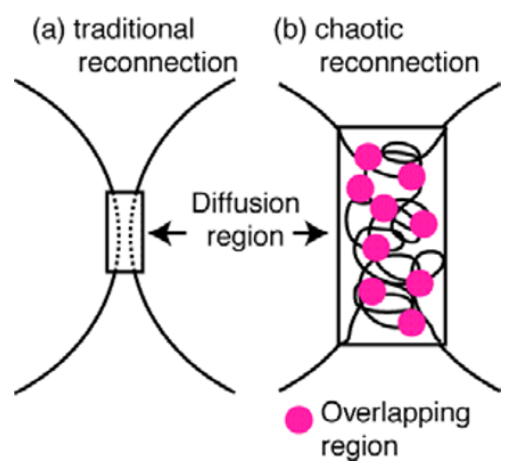

Fig. 6. Illustrations of the diffusion regions are shown. The size of the diffusion region of the chaotic reconnection is relatively large compared with that of the traditional reconnection theory.

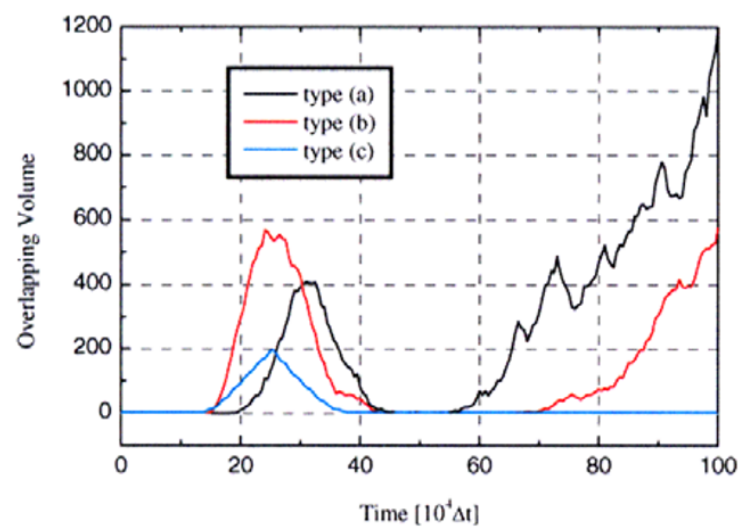

Fig. 7. Evolution of the normalized overlapping volume $\Omega(t, \theta)$ is plotted.

The value of $D(t)$ is usually zero for the case of ideal MHD and positive for the case of non-ideal MHD. It is well known that a kind of dissipation process is needed for the mangetic reconnection. In other words, the factor $D(t)$ should be finite. Thus we introduce an artificial dissipation process, space averaging, which is concerned with $D(t)$. Please note that we do not pay attention to the conventional dissipative reconnection rate here. It is most important that $D(t)$ is not zero but finite.

A new enhancement factor comes from the normalized overlapping volume $\Omega(t, \theta)$. The normalized overlapping 
volume is a mesoscopic enhancement factor. The term mesoscopic means the scale which is describable by MHD but much less than the scale length of the phenomena while the collisionless process is microscopic. We consider that the large $\Omega(t, \theta)$ yields the fast reconnection. Unnormalized overlapping volume is calculated from the time-developed configuration of the filaments numerically and is normalized by the non-chaotic overlapping volume which is determined by the initial configuration. Evolution of the normalized overlapping volume $\Omega(t, \theta)$ is plotted in Fig. 7. For each type, there is a peak at $T=25 \times 10^{4}$ or so, and these peaks are due to the collision. In later time the values of the normalized overlapping volume become large for types (a) and (b), while zero for type (c). This is due to the mixing of the two filaments. The large value of the normalized overlapping volume is rapidly achieved by the chaotic dynamics of the filaments based on the ideal MHD, and means that many overlapping regions emerge everywhere along the tangled filaments. Each overlapping region is small and may be equivalent to the conventional diffusion region. The reconnection rate in this region is determined by $D(t)$. Then the total reconnection rate $R_{\mathrm{f}}(t)$ becomes sufficiently large if the factor $D(t)$ in Eq. (2) has a nonzero value. Thus we conclude that thle chaotic process works to enhance the reconnection rate of the filaments. It is the main mechanism of the chaotic reconnection in the three-dimensional space. If the overlapping volume $\Omega(t, \theta)$ is small, the reconnection time scale is the same as that of the conventional resistive (non-chaotic) reconnection which is determined only by the factor $D(t)$.

\section{Conclusions}

In this work we have proposed a new fast reconnection mechanism, "chaotic reconnection" of the vortex-current filaments in the three-dimensional space. Note that the basic dynamics are based on the nonlinear ideal MHD in which the time scale is mainly determined by the Alfvén transit time, although we have introduced a dissipation process, i.e. threedimensional space averaging. We must extend the present model to realistic one. We shall propose a similar chaotic process as a candidate for the mechanism of the fast magnetic reconnection. Further progress in understanding chaotic reconnection dynamics requires the fully three-dimensional MHD simulations in the high magnetic Reynolds number.

Acknowledgments. This work is supported by Special Postdoctoral Research Program at RIKEN.

\section{References}

Biskamp, D., Collisional and collisionless magnetic reconnection, Phys. Plasmas, 4, 1964-0000, 1997.

Horiuchi, R. and T. Sato, Collisionless magnetic reconnection in the presence of an external driving flow, J. Plasma Phys., 61, 415-000, 1999.

Shibata, K., New observational facts about solar flares from Yohkoh studies, Adv. Space Res., 17, 9-00, 1996.

Yatsuyanagi, Y., T. Hatori, and T. Kato, The equation of motion of vortexcurrent filaments, J. Phys. Soc. Jpn., 65, 745-000, 1996.

Yatsuyanagi, Y., T. Hatori, and T. Kato, Numerical simulations of the vortexcurrent filaments, J. Phys. Soc. Jpn., 67, 166-000, 1998.

Yatsuyanagi, Y., T. Hatori, and T. Kato, Fast mixing mechanism of two vortex-current filaments, J. Plasma Phys., 62, 493-000, 1999.

Y. Yatsuyanagi (e-mail: yyanagi@postman.riken.go.jp), T. Hatori, and T. Kato 